\title{
Mechanisms of survival of protozoan parasites in mononuclear phagocytes
}

\author{
J. MAUEL \\ WHO Laboratories, Institute of Biochemistry, Ch. des Boveresses, \\ CH-1066 Epalinges, Switzerland
}

\begin{abstract}
S U M M A R Y
The understanding of the mechanisms whereby intracellular parasites counteract the microbicidal processes of macrophages has progressed considerably in recent years. Various factors contribute to intracellular parasite destruction; from a biochemical standpoint, particularly important is the oxidative burst triggered by phagocytosis and by macrophage 'activation', that leads to the generation of toxic metabolites of oxygen. At the ultrastructural level, fusion of the parasitophorous vacuole with surrounding lysosomes appears to be a pre-requisite for the final digestion and elimination of the infecting microorganisms. The counter-measures evolved by microorganisms to escape intracellular destruction are best illustrated by studies in vitro on the interaction of parasites of the Leishmania, Toxoplasma and Trypanosoma spp. with mononuclear phagocytes. Some microbes are able to inhibit the fusion of phagosomes with lysosomes, thus avoiding the potentially harmful action of lysosomal hydrolases. Other microorganisms are able to resist the effects of such enzymes, perhaps by secreting inhibitory substances. Others still avoid lysosomes by leaving the phagocytic vacuole, to reach the cytoplasmic matrix where their development is unhindered. Particularly critical is the capacity of certain parasites to subvert the lethal effects of the oxidative burst. This can be achieved either by failing to evoke this metabolic response, or by producing scavengers that can detoxify harmful oxygen metabolites. Intracellular death or survival will thus depend on a delicate balance between the potency of macrophage cidal mechanisms, and the efficacy of the protective measures evolved by the infecting agents.
\end{abstract}

\section{N TRODUCTION}

This short review is concerned with mechanisms whereby certain parasites are able to survive and proliferate within the normally hostile intracellular environment of mononuclear phagocytes. New insights have been gained in this area recently, thanks to detailed ultrastructural and biochemical investigations of the macrophage-parasite interaction. These have uncovered new properties of parasites, particularly as regards their capacity to adapt to living within cells whose very function is to eliminate such intruders. Much of the novel information has been obtained through the study of the infection of mammalian macrophages in vitro by Toxoplasma, Trypanosoma and Leishmania spp. These include parasites that are pathogenic to man: such studies thus represent more than a simple academic exercise; indeed, they are expected to provide fresh leads for the formulation of new therapeutic approaches. 
Before discussing the intracellular defence mechanisms of parasites, however, it is essential that the microbicidal processes of macrophages be briefly examined.

\section{MICROBICIDAL ACTIVITIES OF MACROPHAGES}

\section{(1) Morphological observations}

Excepting those microorganisms that are able to penetrate forcibly within a potential host-cell, internalization of parasites by macrophages is thought to follow the rules established for other particles. Binding to phagocytes triggers contractile elements beneath the segment of plasma membrane adjacent to the contact zone: pseudopods are then formed which invaginate around the particle, following the sequential, circumferential interaction between ligands on the particle and macrophage surface receptors (Griffin, Griffin, Leider \& Silverstein, 1975). Integrity of the macrophage cytoskeletal networks is essential to the phagocytic process, and ingestion of protozoa such as Leishmania mexicana (Alexander, 1975), Leishmania donovani (Chang, 1979), Leishmania tropica* (Ardehali, Khoubyar \& Rezai, 1979) and Trypanosoma cruzi (Nogueira \& Cohn, 1976) by rodent macrophages in vitro is strongly inhibited by the microfilament-depolymerizing agent cytochalasin $B$.

Following ingestion, parasites are enclosed in a vacuole (the phagosome) whose membrane derives from and presumably shares properties with the phagocyte plasma membrane. The morphology of parasitophorous vacuoles appears to vary with both the infecting species and the developmental stage of the parasites, however. Observations on the infection of macrophages in vitro by parasites of the Leishmania genus may serve to illustrate this point. Whereas L. enriettii in mouse and guinea-pig macrophages is usually found within a large vacuole leaving a clear halo around the parasite as seen by light and electron microscopy (Sordat \& Behin, 1977), L. tropica may be enclosed in a tight-fitting vacuole whose trilaminar unit membrane is closely apposed to that of the parasite (Mauel, unpublished observations). After invasion of hamster macrophages by $L$. donovani, both kinds of parasitophorous vacuoles appear to coexist, as well as a third one of mixed type showing parasites partly abutting against the inner lining of the organelle (Chang \& Dwyer, 1978). Ingestion of amastigotes of the $L$. mexicana complex induces characteristically large phagosomes (Benchimol \& De Souza, 1981) in rodent macrophages, and a majority of the parasites appear to be attached to the vacuolar membrane. Freeze-fracture replicas demonstrate an array of intramembranous particles in the parasite plasma membrane presumably adjacent to the phagosome: the role of such structures is, however, unknown (Benchimol \& De Souza, 1981). Morphological differences have also been reported between vacuoles produced in mouse macrophages by infection with promastigotes versus amastigotes of $L$. mexicana, the latter inducing the formation of larger phagosomes (Lewis \& Peters, 1977).

The reasons for these differences, and their possible significance as regards the eventual fate of the intracellular microorganisms, are unknown. Large, distended vacuoles enclosing certain leishmanias might arise from repeated fusion of smaller vesicles with the main vacuole (Glauert, Fell \& Dingle 1969) or from increased intraphagosomal osmotic pressure resulting from the accumulation of indigestible

* The generic name Leishmania tropica has been used instead of Leishmania major in this article, in accordance with the nomenclature used by the authors referred to. 
material (Lewis \& Peters, 1977). In this connexion, it is interesting to note that Leishmania parasites seem to interfere with intracellular digestive processes, perhaps through the release of lysosomal enzyme-inhibiting substances (see page 584).

As shown by electron or light microscopy using appropriate cytological markers, microbe-containing phagosomes usually undergo fusion with primary or secondary lysosomes, which contain hydrolytic enzymes required for the degradation of foreign materials. Fusion of the two vesicles presumably constitutes an important step in the destruction of infectious agents, and indeed, lysosome-phagosome fusion is generally associated with the killing of intracellular microbes. As discussed below, however, important exceptions to this rule have been documented. Moreover, it has to be emphasized that digestion by lysosomal enzymes presumably constitutes, in most instances, a late step in the elimination of intracellular pathogens, which requires a preliminary 'lethal hit' usually provided by -toxic metabolites of oxygen.

\section{(2) Biochemical events}

Ingestion of particles (including live or dead protozoa) by professional phagocytes tends to induce in the latter cells a chain of metabolic events aimed at the destruction of invading pathogens, and referred to as the 'respiratory burst'. This metabolic activity is characterized by an increased uptake of oxygen and the rapid oxidation of glucose through the hexose monophosphate shunt (HMPS) pathway (Babior, 1978; Johnston, 1978). The phagocytosis-driven respiratory burst appears to be initiated by the stimulation of a plasma membrane-bound oxidase accepting reduced pyridine nucleotides as electron donors (Forman, Nelson \& Fischer, 1980; Bellavite, Breton, Dri \& Soranzo, 1981), and catalysing the univalent reduction of molecular oxygen to form the superoxide anion $\left(\mathrm{O}_{2}^{-}\right)$. Various metabolites of oxygen that are toxic for microorganisms may then be formed. Dismutation of superoxide yields hydrogen peroxide $\left(\mathrm{H}_{2} \mathrm{O}_{2}\right)$; formation of the hydroxyl radical ( $\mathrm{HO}^{\circ}$ ) and of singlet oxygen $\left({ }^{1} \mathrm{O}_{2}\right)$ may then proceed by interaction of the former two molecular species (Badwey \& Karnovsky, 1980). HMPS stimulation is explained by the reoxidation of reduced nicotinamide-adenine-dinucleotidephosphate (NADPH), an essential coenzyme of this metabolic pathway. Such reoxidation may occur either directly through the above oxidase system, or indirectly by $\mathrm{H}_{2} \mathrm{O}_{2}$ via a glutathione-peroxidase loop (Weidemann, Smith, Haeney \& Alaudeen 1980). It may be noted that since the enzymic machinery participating in the generation of oxygen derivatives is part of the phagocyte plasma membrane it is incorporated in the membrane of the parasitophorous vacuole during phagocytosis. Oxygen metabolites are thus presumably delivered in the direct vicinity of the microorganisms to be destroyed.

The above oxidative processes are particularly marked in activated macrophages. This term refers to a particular physiological state of macrophages, which may arise as the consequence of an immune response of the cell-mediated type or through other mechanisms (Mauel, 1982), and whereby the cells display an increased capacity to destroy intracellular pathogens. Such enhanced microbicidal capacity has been clearly linked to a stimulation of the oxidative metabolic response of macrophages resulting from activation (Murray \& Cohn, 1980; Buchmüller \& Mauel, 1981). 


\section{(3) Microbicidal mechanisms}

The microbicidal mechanisms of macrophages are usually divided into those dependent and those not dependent on the presence of oxygen. The following observations support the hypothesis that oxygen metabolites generated during the respiratory burst constitute the most potent anti-microbial agents available to macrophages. (a) Intracellular destruction of Leishmania and Toxoplasma spp. upon phagocytosis by normal macrophages correlates with stimulation of the respiratory burst and conversely, failure to trigger such metabolic activity is linked with intracellular survival (Wilson, Tsai \& Remington, 1980; Murray, 1981 $a, b$; Haidaris \& Bonventre, 1982). (b) Killing of Leishmania parasites by activated macrophages is concomitant with the development of oxidative phenomena, and the production of $\mathrm{H}_{2} \mathrm{O}_{2}$ by various classes of macrophages closely correlates with their capacity to destroy T. cruzi (Nathan, Nogueira, Juangbhanich, Ellis \& Cohn, 1979; Buchmüller \& Mauel, 1981 ; Haidaris \& Bonventre, 1982). (c) Incubation of macrophages with scavengers of oxygen metabolites reduces their parasiticidal activity (Murray, Juangbhanich, Nathan \& Cohn, 1979; Buchmüller \& Mauel, 1981 ; Haidaris \& Bonventre, 1982). (d) The capacity of microorganisms to survive intracellularly correlates well with their natural endowment in scavengers of oxygen metabolites (Murray \& Cohn, 1979 ; Murray, $1981 a$ ). Furthermore, parasites are rapidly destroyed in vitro when incubated in cell-free preparations containing $\mathrm{H}_{2} \mathrm{O}_{2}$, peroxidase and a halide, a system originally described by Klebanoff (1967) (Buchmüller \& Mauel, 1981). The precise mechanisms of intracellular killing by products of the respiratory burst are still incompletely determined. They may involve the peroxidation of surface lipids and the decarboxylation of amino acids yielding toxic aldehydes, as well as the induction of chromosomal damage (Badwey \& Karnovsky, 1980).

Toxic mechanisms other than the metabolic generation of oxygen derivatives have been described in phagocytic cells. Those regarded as potentially important in bacterial killing are acid production, membrane hydrolysis by lysosomal enzymes, digestion of microbial capsules by lysozyme, chelation of iron by iron-binding proteins and membrane labilization by cationic peptides (Klebanoff, 1980). None of these mechanisms has been shown yet to be of significance in the destruction of intracellular parasitic protozoa.

INTRACELLULAR PARASITE SURVIVAL

A number of parasitic protozoa are able to survive and multiply within phagocytes; their capacity to resist microbicidal processes undoubtedly plays a role in their pathogenicity. Two types of mechanisms have been documented that appear to contribute to intracellular survival: (1) the ability to withstand or to circumvent the hydrolytic activity of lysosomes and (2) the capacity to inhibit the respiratory burst, or to destroy toxic compounds generated by macrophage oxidative metabolism. 


\section{(1) Resistance to lysosomal toxicity}

The relationship established by parasites with intracellular organelles can be conveniently studied in vitro by light and electron microscopy using endogenous and exogenous lysosomal markers such as acid phosphatase, acridine orange, thorium oxide and other electron-dense substances, or horseradish peroxidase (Alexander \& Vickerman, 1975; Ebert, Enriquez \& Mühlpfordt, 1976; Lewis \& Peters, 1977; Chang \& Dwyer, 1978). In this way, at least three types of mechanism have been recognized, whereby parasites may avoid the harmful effects of lysosomal constituents.

\section{(a) Inhibition of phagosome-lysosome fusion}

Through mechanisms that remain unclear, certain pathogens appear to be capable of preventing the phagocytic vacuole from undergoing fusion with surrounding lysosomes, thus presumably avoiding contact with potentially harmful lytic enzymes. Thus, morphological evidence suggests that Toxoplasma gondii alters the properties of the phagosomes, allowing the apposition of strips of endoplasmic reticulum and mitochondria along the vacuolar membrane, and perhaps hindering in this way the access of lysosomes to the parasitophorous vacuole. A similar situation has been described in the infection of mouse macrophages in vitro by virulent Mycobacterium tuberculosis (Armstrong \& D'Arcy Hart, 1971). This behaviour depends on the integrity of the microorganisms: parasites that have been damaged by physical means, for example heat, or coated with polyclonal or monoclonal specific antibody, display a lesser capacity to prevent phagolysosome formation (Armstrong \& D'Arcy Hart, 1975; Sethi, Eudo \& Brandis, 1981), suggesting that the phenomenon depends on the metabolic activity and/or surface characteristics of the live, intact parasite.

The mechanisms of inhibition of phagosome-lysosome fusion are unknown. It has been suggested that polyanionic substances, such as the acidic sulphatides of the mycobacterial cell wall, may contribute to fusion blockade (Goren, D'Arcy Hart, Young \& Armstrong, 1976). Moreover, fusion inhibition could be obtained by incubation of macrophages in vitro with poly-D-glutamic acid (Alexander, 1981). The relevance of these observations to the mechanisms of parasite-mediated fusion inhibition is unclear, however. Indeed, another natural polyanionic compound, the leishmanial excreted factor, appears to display no inhibitory activity on phagosomelysosome fusion, since Leishmania parasites which secrete this factor (Schnur, Zuckerman \& Greenblatt, 1972; Slutzky \& Greenblatt, 1979) do reside in phagolysosomes (Alexander \& Vickerman, 1975 ; Chang \& Dwyer, 1976). As reported by Gordon, D'Arcy Hart \& Young (1980), extraneously added ammonia also strongly inhibits fusion between phagosomes and lysosomes in Saccharomyces cerevisiaeinfected mouse peritoneal macrophages, as well as inducing lysosome swelling; both phenomena are reversible upon removal of the base. Interestingly, other cationic substances such as chloroquine and tributylamine display an opposite effect, i.e. they enhance phagolysosome formation (D'Arcy Hart \& Young, 1978). As both ammonia and the above amines accumulate in lysosomes, where they raise the $\mathrm{pH}$ and appear to interfere with protein degradation (Ohkuma \& Poole, 1978; Seglen, Grinde \& Solheim, 1979), it is unlikely that the opposite effects on fusion 
are mediated by $\mathrm{pH}$ changes or by an alteration in intralysosomal protein metabolism.

The importance as a survival mechanism of the capacity of certain parasites to inhibit phagosome-lysosome fusion is uncertain. In most instances the precise contribution of phagosome-lysosome fusion to intracellular parasite killing, relative to that of other cidal mechanisms, is difficult to assess. For example, as noted above, coating $T$. gondii with specific antibody (which does not by itself harm the parasite) promotes the fusion of lysosomes with the parasitophorous vacuole, as well as rendering this normally resistant microorganism fully susceptible to intracellular killing by normal macrophages. Since such treatment of the target organism also stimulates the oxidative burst and the subsequent generation of toxic oxygen metabolites by phagocytosing macrophages (Wilson et al. 1980), it remains to be determined whether phagosome-lysosome fusion is essential to parasite killing or whether it should merely be regarded as a late event aimed at digesting an already dead parasite. The same type of reasoning holds in the case of the inverse correlation reported between the virulence of three strains of Nocardia asteroides and the degree of phagosome-lysosome fusion following internalization by rabbit alveolar macrophages (Davis-Scibienski \& Beaman, 1980).

Experiments relating the level of phagolysosome formation and intracellular parasite multiplication suggest, however, that even for parasites regarded as natural inhabitants of lysosomes the lysosomal milieu may constitute a suboptimal environment. Thus macrophage treatment with poly-D-glutamic acid both inhibited the fusion of lysosomes with macrophage vacuoles containing $L$. mexicana mexicana and promoted intracellular parasite growth relative to that in untreated controls (Alexander, 1981). This effect was particularly marked when macrophages were infected with promastigotes as opposed to amastigotes, further suggesting that transformation to amastigotes constitutes an adaptative response of the parasite to the harshness of intralysosomal living (Lewis \& Peters, 1977).

\section{(b) Resistance to lysosomal enzymes}

Studies on the interaction of Leishmania with host macrophages indicate that the intracellular 'niche' of this parasite is the phagolysosome itself. Thus, using endogenous or exogenous markers to follow the fate of the intracellular parasite at the ultrastructural level, several investigators using parasites from different species in various host macrophages have shown that the parasitophorous vacuole fuses with primary and secondary lysosomes, apparently without major harm to the parasite (Alexander \& Vickerman, 1975 ; Chang \& Dwyer, 1976; Ebert et al. 1976; Berman, Dwyer \& Wyler, 1979). The demonstration that extracellularly added electron-dense material is eventually detectable around parasites in their intracellular location indicates a continuous accessibility to the extracellular environment via the vacuolar apparatus of the host-cell, a finding perhaps significant in terms of parasite nutrition and chemotherapy (Shepherd, Stahl, Bernd \& Rabinovitch, 1983).

Amastigotes of Leishmania parasites appear not to be seriously affected by the intralysosomal milieu and can proliferate in such an environment. This resistance may be only partial, however, since as noted above, intracellular parasite growth 
is promoted by treatment of the macrophages with fusion-inhibiting agents (see page 583). Moreover, Leishmania promastigotes appear more susceptible to intracellular degradation than amastigotes; indeed, it has been proposed that survival of ingested promastigotes may depend on their capacity to transform rapidly to amastigotes (Lewis \& Peters, 1977). Of relevance in this context is the observation that promastigotes of $L$. donovani can infect human fibroblasts in vitro, in which cells' transformation to amastigotes occurs in the absence of phagolysosome fusion, i.e. in an environment perhaps less detrimental to the parasite (Chang, 1978).

The mechanisms of intralysosomal survival are obscure, and evidence sometimes contradictory. Inert, non-digestible material such as the lipidic capsule encasing Mycobacteria may protect these organisms from attack by lysosomal hydrolases (Draper \& Rees, 1970). No such protective coating is available to leishmanias, however. When the $\mathrm{pH}$ of $L$. donovani- and $L$. mexicana amazonensis-infected parasitophorous vacuoles in cells of the murine macrophage line J774G8 was determined using a fluorescent probe, it was found to be in the range $5 \cdot 0-5 \cdot 7$, i.e. no different from that of non-infected cells (Chang, 1980). Yet, amastigotes of L. mexicana mexicana appear to release large amounts of ammonia (Coombs, G. H. \& Alexander, J. A., personal communication). Other observations support the idea that lysosomal enzyme activity remains unimpaired in the presence of the parasite: as shown by Chang \& Dwyer (1978), host vacuolar membrane tightly adhering to amastigotes may be carried over during isolation of the parasite from infected tissue and remain bound to the parasite after ingestion by new macrophages in vitro. Once the parasite has reached the intralysosomal compartment, however, this material is rapidly digested, suggesting that lysosomal enzymes remain active. As the parasite survives undamaged, however, it has been proposed that its membrane itself is 'refractory' to lysosomal degradation (Chang \& Dwyer, 1978).

Other evidence indicates that Leishmania parasites do inhibit lysosomal enzyme activity, however. Thus, pre-infection of hamster macrophages with virulent L. donovani enabled the phagocytes to retain significantly more Leptomonas costoris than did control, non-infected cells or cells infected with an avirulent strain of L. donovani (Kutish \& Janovy, 1981). These observations suggest that leishmanial infection might alter the overall digestive functions of macrophages, and that the ability to effect such alterations might be related to parasite virulence. They are consistent with the hypothesis that leishmanias might survive in phagolysosomes by interfering with lysosomal digestive capacity, possibly through the release of enzyme-inhibiting substances. In this context, Leishmania parasites have been shown to synthesize in vivo and in vitro a carbohydrate-containing material, the excreted factor (EF) (see page 583), that is endowed with anti- $\beta$-galactosidase properties (El-On, Bradley \& Freeman, 1980). EF is negatively charged, as are other inhibitors of lysosomal enzymes such as heparin or chondroitin-sulphate, which are thought to function as moderators of leucocytic lysosomal activity (Avila \& Convit, 1976). That such compounds may protect parasites against intracellular degradation is suggested by the demonstration that growth of $L$. enriettii in otherwise non-permissive host macrophages was promoted by the addition of exogenous EF (Handman \& Greenblatt, 1977).

In addition to the above, amastigotes of $L$. mexicana have been reported recently to contain several-fold higher proteinase activity than promastigotes, macrophages 
or other flagellate protozoa (Coombs, 1982). It was suggested that such proteinases, if secreted by the amastigote, could inactivate host lysosomal hydrolases and so decrease the macrophage microbicidal potency. Further information relating parasite virulence to its proteinase activity is awaited with interest.

\section{(c) Escape from the phagolysosomes}

As demonstrated by electron microscopy, certain pathogens appear capable of escaping from the intravacuolar spaces, presumably by disrupting the phagolysosomal membrane, to reach the cytoplasmic matrix where their multiplication can proceed unhindered. So do a number of viruses and, possibly, Mycobacterium leprae (Mauel, 1982). In studies of the interaction in vitro of T. cruzi with mouse macrophages, Tanowitz, Wittner, Kress \& Bloom (1975) reported that the parasites were usually located within phagocytic vacuoles, although occasionally they came to lie in the host-cell cytoplasm, as evidenced by the fact that these microorganisms were not visibly surrounded by two sets of membrane. These results were extended by Nogueira \& Cohn (1976), who concluded that uptake of both epimastigotes and trypomastigotes was by phagocytosis. In each instance the organisms were initially surrounded by a plasma membrane-derived phagosome. Striking differences could then be observed, depending on the developmental stage of the parasite. Whereas epimastigotes were killed and digested within phagolysosomes, trypomastigotes and transition forms were reported to escape from the phagocytic vacuole, then to multiply in the cytoplasmic matrix. It is noteworthy that, when taken up by activated macrophages, trypomastigotes were then easily destroyed in the phagolysosomes, suggesting that the parasites had been killed before they had a chance to reach a safer intracellular location (Kress, Bloom, Wittner, Rowen \& Tanowitz, 1975).

\section{(2) Parasite defences against intracellular killing: metabolic aspects}

As noted earlier, phagocytosis and activation are accompanied by a stimulation of the macrophages (see page 581). As a consequence, metabolites of oxygen are generated that appear to constitute one of the main groups of microbicidal molecules produced by phagocytes. Metabolic studies on macrophages ingesting different types of particles, as well as investigations into parasite biochemistry, have uncovered two mechanisms that enable microorganisms to circumvent such toxic processes: (1) the capacity to be ingested without stimulating a respiratory burst and (2) the neutralization of toxic oxygen derivatives.

\section{(a) Phagocytosis without oxidative burst}

Whereas certain classes of phagocytes, including normal human monocytes and activated human or mouse macrophages, can destroy or otherwise inhibit ingested T. gondii, non-activated macrophages are unable to do so (Wilson et al. 1980). Defective intracellular killing appears to be linked with the capacity of the parasite to be phagocytosed without stimulating a respiratory burst. Thus, ingestion of $T$. gondii by resident mouse peritoneal macrophages in vitro failed to elicit the reduction of nitro-blue tetrazolium (Murray, 1981 a), or to produce chemilumi- 
nescence and stimulate the oxidation of glucose through the hexose shunt pathway (Wilson et al. 1980), compared with the phagocytosis of other types of particles such as Candida and Leishmania spp. The reasons for this behaviour are unknown. They may depend on characteristics of the parasite surface or on the type of macrophage receptors that are involved in phagocytosis. Indeed, as discussed above (see page 583), coating $T$. gondii with specific antibody rendered the parasite fully susceptible to intracellular killing by mouse macrophages (Sethi et al. 1981) as well as allowing the phagocytosis-driven respiratory burst to be generated (Wilson et al. 1980). Whether the in vitro susceptibility of antibody-coated toxoplasmas to intracellular destruction may explain the partial protection afforded by the in vivo transfer of immune serum is uncertain, however, (Hafizi \& Moddaber, 1978).

The critical importance as a survival strategy of the capacity to avoid triggering the respiratory burst has been further emphasized in other experimental systems. As shown by Murray $(\mathbf{1 9 8 1} b)$, cells of a murine macrophage line that are deficient in their oxidative metabolism did ingest but failed to destroy the normally highly susceptible promastigotes of L.donovani. As discussed earlier, intracellular survival of Leishmania parasites may depend on their ability to transform from promastigotes to amastigotes. It was thus of interest to compare both stages of parasite development with respect to their capacity to initiate a metabolic response in macrophages. Indeed, amastigotes of $L$. donovani failed to provide the stimulus necessary to elicit the oxidative burst in rodent macrophages, whether normal or pre-activated (Haidaris \& Bonventre, 1982); consequently their intracellular survival was good. Under the same conditions promastigotes did trigger a strong metabolic response and were destroyed (Murray, 1981 a). This characteristic of the amastigote is not reversed by precoating with antibody or by heat-killing; it appears to constitute an intrinsic property of the amastigote surface, and may explain the greater infectivity of amastigotes for experimental animals compared with infectivity of the promastigote form.

\section{(b) Neutralization of oxygen metabolites}

Intracellular protozoa display a wide spectrum of susceptibility to toxic substances generated during the macrophage oxidative burst; to a certain extent, such differences are reflected in the contrasting fates of various parasites engulfed by macrophages. Thus, in a comparative study of the interaction of mouse peritoneal macrophages with Leishmania spp. and T. gondii, Murray (1981a) observed that $L$. tropica and $L$. donovani promastigotes were both destroyed within $18 \mathrm{~h}$ of being phagocytosed. Parasite killing was, as expected, concomitant with triggering of the macrophage oxidative burst and production of $\mathrm{H}_{2} \mathrm{O}_{2}$ by the phagocytes. The observation that catalase partially inhibited the leishmanicidal activity of the macrophages suggested that the metabolically generated $\mathrm{H}_{2} \mathrm{O}_{2}$ was sufficient for killing these microorganisms. It was shown also that Leishmania parasites are poor in endogenous catalase and glutathione-peroxidase, two scavengers of hydrogen peroxide. In contrast $T$. gondii, which fails to trigger the respiratory burst (see page 586), was also found to be more resistant to $\mathrm{H}_{2} \mathrm{O}_{2}$, presumably because this microorganism is richly endowed with catalase and 
glutathione-peroxidase. These results suggest that the level of endogenous scavengers of oxygen metabolites also plays a crucial role in determining the susceptibility of parasites to killing within phagocytic cells.

\section{THE FATE OF INTRACELLULAR MICROORGANISMS AS DETERMINED BY} THE BALANCE BETWEEN CIDAL AND DEFENCE FACTORS

It is thus evident that the fate of a microorganism interiorized by macrophages will depend on a balance between several factors. If the microorganism is to survive, its nutritional and physico-chemical requirements must obviously be satisfied by the host-cell (not discussed in this review). In addition, the ability (or lack of it) of macrophages to target lethal compounds or otherwise create conditions aimed at destroying the invading microbe, as well as the aptitude of the latter to evade or counteract such processes, will determine the eventual outcome of infection. Experience shows such balance to be very unpoised; in other terms, the varying behaviours detected in vitro in different host cell-parasite combinations usually reflect quantitative rather than truly qualitative differences, and may often be modified by changing the experimental conditions. Hence, results obtained from in vitro studies do not always translate easily into the in vivo situation.

Thus it is essential to realize that macrophages may function as host-cells even for parasites whose phagocytosis does trigger a respiratory burst. For instance, as discussed above, mouse peritoneal macrophages produce a strong metabolic response upon promastigotes of $L$. donovani and $L$. tropica; in addition, these parasites contain little catalase and glutathione-peroxidase to protect them from attack by hydrogen peroxide. Such conditions lead to in vitro parasite killing.

Yet it is obvious that both microorganisms can infect mice. Moreover, provided infection ratio and duration are adequate, both parasites will eventually also become established in murine macrophages in vitro. The same situation prevails in the case of infection of human macrophages by $L$. donovani promastigotes: the phagocytes become infected in spite of their producing a strong oxidative burst in response to parasite challenge (Pearson, Harcus, Symes, Romito \& Donowitz, 1982). Thus the capacity of macrophages to resist leishmanial infection can be overcome easily under both in vivo and in vitro conditions, presumably by exhaustion of the macrophage cidal mechanisms and the acquisition by the parasites, through conversion to the amastigote form, of a higher survival potential.

Once the parasite is established intracellularly, new factors may come into play that will weigh heavily on the fate of the microorganism. A major element of host defence is the capacity to respond to parasite antigens by mounting an immune response that may lead to macrophage activation. In this instance also, however, differences can be detected in vitro between various host-parasite combinations; these appear to constitute a determining factor in the overall host susceptibility to infection. Thus mice are completely resistant to infection by $L$. enriettii; this correlates with the observation that activated macrophages from this host species readily destroy the parasite in vitro (Behin, Mauel, Biroum-Noerjasin \& Rowe, 1975; Mauel, Buchmüller \& Behin, 1978). A similar parallel can be found in the 
infection of mice by $L$. tropica; genetically determined variations can be detected between mice from different inbred strains as regards their susceptibility to infection by this parasite (Preston \& Dumonde, 1976; Behin, Mauel \& Sordat, 1979; Handman, Ceredig \& Mitchell, 1979). Whereas 'susceptible' animals tend to carry infection for their lifetime, 'resistant' ones do produce a skin infection at first, then succeed in eliminating the pathogen, presumably through an immune response leading to macrophage activation. In vitro studies have shown that activated macrophages from 'resistant' hosts are more efficient at killing the parasite than are activated macrophages from susceptible ones (Behin et al. 1979). It is important to note, however, that the deficiency observed in activated 'susceptible' macrophages is only partial: indeed, under culture conditions favouring maximal activation, susceptible macrophages (with respect to $L$. tropica infection) are able to eliminate the parasite completely. Relatively modest qualitative variations that are detectable in vitro, may thus translate into major in vivo differences as regards susceptibility or resistance to a given infectious agent.

The author gratefully acknowledges financial support from the Swiss National Federation (Grant 3.249-0.82) and from the Leishmaniasis component of the UNDP/World Bank/WHO Special Programme for Research and Training in Tropical Diseases.

\section{REFERENCES}

Alexander, J. (1975). Effect of the antiphagocytic agent Cytochalasin B on macrophage invasion by Leishmania mexicana promastigotes and Trypanosoma cruzi epimastigotes. Journal of Protozoology 22, 237-40.

AleXANDer, J. (1981). L. mexicana: Inhibition and stimulation of phagosome-lysosome fusion in infected macrophages. Experimental Parasitology 52, 261-70.

Alexander, J. \& Vickerman, K. (1975). Fusion of host cell secondary lysosomes with the parasitophorous vacuole of L. mexicana-infected macrophages. Journal of Protozoology 22, $502-8$.

Ardehali, S. M., Khovbyar, K. \& Rezat, H. R. (1979). Studies on the effect of the antiphagocytic agent Cytochalasin B on Leishmania-macrophage interaction. Acta Tropica 36, 15-19.

Armstrone, J. A. \& D'Arcy Hart, P. (1971). Response of cultured macrophages to Mycobacterium tuberculosis, with observations on fusion of phagosomes with lysosomes. Journal of Experimental Medicine 134, 713-40.

Armstrong, J. A. \& D'Arcy Hart, P. (1975). Phagosome-lysosome interactions in cultured macrophages infected with virulent tubercle bacilli. Reversal of the usual non-fusion pattern and observations on bacterial survival. Journal of Experimental Medicine 142, 1-16.

Avila, J. L. \& Convit, J. (1976). Physicochemical characteristics of the glycosamino-glycanlysosomal enzyme interaction in vitro. The Biochemical Journal 160, 129-36.

BABIOR, B. M. (1978). Oxygen-dependent microbial killing by phagocytes. New England Journal of Medicine 298, 659-68, 721-5.

BaDWEY, J. A. \& KaRnovsky, M. L. (1980). Active oxygen species and the function of phagocytic leukocytes. Annual Review of Biochemistry 49, 695-726.

Behin, R., Mauel, J., Biroum-Noerjasin \& Rowe, D. S. (1975). Mechanisms of protective immunity in experimental cutaneous leishmaniasis of the guinea-pig II. Selective destruction of different Leishmania species in activated guinea-pig and mouse macrophages. Clinical and Experimental Immunology 20, 351-60.

Behin, R., Mauel, J. \& Sordat, B. (1979). Leishmania tropica: pathogenicity and in vitro macrophage function in strains of inbred mice. Experimental Parasitology 48, 81-91.

Bellavite, P., Breton, G., Dri, P. \& Soranzo, R. (1981). Enzymatic basis of the respiratory burst of guinea-pig resident peritoneal macrophages. Journal of the Reticuloendothelial Society 29, 47-60.

Benchimol, M. \& De Sovza, W. (1981). Leishmania mexicana amazonensis: attachment to the 
membrane of the phagocytic vacuole of macrophages in vitro. Zeitschrift für Parasitenkunde 66, 25-9.

Berman, J. D., Dwyer, D. M. \& Wyler, D. J. (1979). Multiplication of Leishmania in human macrophages in vitro. Infection and Immunity 26, 375-9.

Buchmüller, Y. \& MAUkL, J. (1981). Studies on the mechanisms of macrophage activation: possible involvement of oxygen metabolites in killing of Leishmania enriettii by activated mouse macrophages. Journal of the Reticulo-endothelial Society 29, 181-92.

Chane, K. P. (1978). Leishmania infection of human skin fibroblasts in vitro: absence of phagolysosomal fusion after induced phagocytosis of promastigotes, and their intracellular transformation. American Journal of Tropical Medicine and Hygiene 27, 1084-96.

Chang, K. P. (1979). Leishmania donovani: promastigote-macrophage surface interactions in vitro. Experimental Parasitology 48, 175-89.

Chang, K. P. (1980). Endocytosis of Leishmania-infected macrophages. Fluorometry of pinocytic rate, lysosome-phagosome fusion and intralysosomal $\mathrm{pH}$. In The Host-Invader Interplay (ed. H. Van den Bossche), pp. 231-4. Amsterdam: Elsevier-North Holland.

Chang, K. P. \& Dwyer, D. M. (1976). Multiplication of a human parasite (Leishmania donovani) in phagolysosomes of hamster macrophages in vitro. Science 193, 678-80.

ChAng, K. P. \& Dwyer, D. M. (1978). Leishmania donovani-hamster macrophage interactions in vitro: cell entry, intracellular survival and multiplication of amastigotes. Journal of Experimental Medicine 147, 515-30.

Coombs, G. H. (1982). Proteinases of Leishmania mexicana and other flagellate protozoa. Parasitology 84, 149-55.

D'Arcy Hart, P. \& Young, M. R. (1978). Manipulation of the phagosome-lysosome fusion response in cultured macrophages. Enhancement of fusion by chloroquine and other amines. Experimental Cell Research 114, 486-90.

Davis-Scibienski, C. \& Beaman, B. L. (1980). Interaction of Nocardia asteroides with rabbit alveolar macrophages: association of virulence, viability, ultrastructural damage, and phagosome-lysosome fusion. Infection and Immunity 28, 610-19.

Draper, P. \& ReEs, R. J. W. (1970). Electron-transparent zone of mycobacteria may be a defence mechanism. Nature, London 228, 860-1.

Ebert, F., Enriquez, G. L. \& MÜhlPfordt, H. (1976). Electron microscopic studies of the phagocytosis of Leishmania donovani by hamster peritoneal macrophages and its lysosomal activity in vivo. Behring Institut Mitteilungen 60, 65-74.

El-ON, J., Bradley, D. J. \& Freeman, J. C. (1980). Leishmania donovani: action of excreted factor on hydrolytic enzyme activity of macrophages from mice with genetically different resistance to infection. Experimental Parasitology 49, 167-74.

Forman, H. J., Nelson, J. \& Fischer, A. B. (1980). Rat alveolar macrophages require NADPH for superoxide production in the respiratory burst. Journal of Biological Chemistry 255, 9879-83.

Glauert, A. M., Fell, H. B. \& Dingle, J. T. (1969). Endocytosis of sugars in embryonic skeletal tissues in organ culture. II. Effect of sucrose on cellular fine structure. Journal of Cell Science 4, 105-31.

Gordon, A. H., D'Arcy Hart, P. \& Young, M. R. (1980). Ammonia inhibits phagosome-lysosome fusion in macrophages. Nature London 286, 79-80.

Goren, M. B., D'Arcy Hart, P., Young, M. R. \& Armstrong, J. A. (1976). Prevention of phagosome-lysosome fusion in cultured macrophages by sulfatides of Mycobacterium tuberculosis. Proceedings of the National Academy of Sciences, USA 73, 2510-14.

Griffin, F. M., Griffin, J. A., Leider, J. E. \& Silverstein, S. C. (1975). Studies on the mechanisms of phagocytosis. Journal of Experimental Medicine 142, 1263-82.

HAFizi, A. \& ModdaBer, F. Z. (1978). Effect of cyclophosphamide on Toxoplasma gondii infection : reversal of the effect by passive immunization. Clinical and Experimental Immunology 23, 389-94.

Haidaris, C. G. \& Bonventre, P. F. (1982). A role for oxygen-dependent mechanisms in killing of Leishmania domovani tissue forms by activated macrophages. Journal of Immunology 129, $850-5$.

Handman, E. \& Greenblatt, C. L. (1977). Promotion of leishmanial infection in non-permissive host-macrophages by conditioned medium. Zeitschrift für Parasitenkunde 53, 143-7.

Handman, E., Ceredia, R. \& Mitcheld, G. F. (1979). Murine cutaneous leishmaniasis: disease patterns in intact and nude mice of various genotypes and examination of some differences 
between normal and infected macrophages. Australian Journal of Experimental Biology and Medical Sciences 57, 9-30.

Johnston, R. B. (1978). Oxygen metabolism and the microbicidal activity of macrophages. Federation Proceedings 37, 2759-64.

Kress, Y., Bloom, B. R., WitTner, M., Rowen, A. \& Tanowitz, H. (1975). Resistance of Trypanosoma cruzi to killing by macrophages. Nature, London 257, 394-6.

Kutish, G. F. \& JAnovy, J. (1981) Inhibition of in vitro macrophage digestion capacity by infection with Leishmania donovani. Journal of Parasitology 67, 457-62.

Lewis, D. H. \& Peters, W. (1977). The resistance of intracellular Leishmania parasites to digestion by lysosomal enzymes. Annals of Tropical Medicine and Parasitology 71, 295-312.

MaUel, J. (1982). Effector and escape mechanisms in host-parasite relationships. In Progress in Allergy, vol. 31 (ed. P. Kallos), pp. 1-75. Basel: Karger.

Mauel, J., Buchmüller, Y. \& Behin, R. (1978). Destruction of intracellular Leishmania enriettii in macrophages activated by cocultivation with stimulated lymphocytes. Journal of Experimental Medicine 148, 393-407.

Murray. H. W. (1981 a). Susceptibility of Leishmania to oxygen intermediates and killing by normal macrophages. Journal of Experimental Medicine 153, 1302-15.

Murray, H. W. (1981 b). Interaction of Leishmania with a macrophage cell line. Journal of Experimental Medicine 153, 1690-5.

Murray, H. W. \& Cohn, Z. A. (1979). Macrophage oxygen-dependent antimicrobial activity. I. Susceptibility of Toxoplasma gondii to oxygen intermediates. Journal of Experimental Medicine 150, 938-49.

Murray, H. W. \& Cohn, Z. A. (1980). Macrophage oxygen-dependent antimicrobial activity. III. Enhanced oxidative metabolism as an expression of macrophage activation. Journal of Experimental Medicine 152, 1596-609.

Murray, H. W., Juangbhanich, C. W., Nathan, C. F. \& Cohn, Z. A. (1979). Macrophage oxygen-dependent antimicrobial activity. II. The role of oxygen intermediates. Journal of Experimental Medicine 150, 950-64.

Nathan, C. F., Nogueira, N., Juangbhanich, C. W., Ellis, J. \& Cohn, Z. A. (1979). Activation of macrophages in vivo and in vitro. Correlation between hydrogen peroxide release and killing of Trypanosoma cruzi. Journal of Experimental Medicine 149, $1056-68$.

Nogueira, N. \& CoHn, Z. A. (1976). Trypanosoma cruzi: mechanism of entry and intracellular fate in mammalian cells. Journal of Experimental Medicine 143, 1402-20.

Oнкuma, S. \& Poole, B. (1978). Fluorescence probe measurement of the intralysosomal pH in living cells and the perturbation of $\mathrm{pH}$ by various agents. Proceedings of the National Academy of Sciences, USA 75, 3327-31.

Pearson, R. L., Harcus, J. L., Symes, P. H., Romito, R. \& Donowitz, G. R. (1982). Failure of the phagocytic oxidative response to protect human monocyte-derived macrophages from infection by Leishmania donovani. Journal of Immunology 129, 1282-6.

Preston, P. M. \& Dumonde, D. C. (1976). Immunology of clinical and experimental Leishmaniasis. In Immunology of Parasitic Infections (ed. S. Cohen and E. H. Sadun), pp. 167-202. Oxford: Blackwell Scientific Publications.

Schnur, L. F., Zuckerman, A. \& Greenblat, C. L. (1972). Leishmanial serotypes as distinguished by gel diffusion of factors excreted in vitro and in vivo. Israel Journal of Medical Sciences 8, 932-42.

Seglen, P. O., Grinde, B. \& Solheim, A. E. (1979). Inhibition of the lysosomal pathway of protein degradation in isolated rat hepatocytes by ammonia, methylamine, chloroquine and leupeptin. European Journal of Biochemistry 95, 215-25.

Sethi, K. K., Eudo, T. \& Brandis, H. (1981). Toxoplasma gondii trophozoites precoated with specific monoclonal antibodies cannot survive within normal murine macrophages. Immunology Letters 2, 343-6.

Shepherd, V. L., Stahl, P. D., Bernd, P. \& Rabinovitch, M. (1983). Receptor-mediated entry of $\beta$-glucuronidase into the parasitophorous vacuoles of macrophages infected with Leishmania mexicana amazonensis. Journal of Experimental Medicine 157, 1471-82.

SluTzKy, G. M. \& GreenblatT, C. L. (1979). Analysis by SDS-polyacrylamide gel electrophoresis of an immunologically active factor of Leishmania tropica from growth media, promastigotes and infected macrophages. Biochemical Medicine 21, 70-7.

Sordat, B. \& BeHIN, R. (1977). Cutaneous leishmaniasis of the guinea pig: a sequential study by light and electron microscopy. In Ecologie des Leishmanioses, pp. 87-93. Paris: INSERM. 
Tanowitz, H., Wittner, M., Kress, Y. \& Bloom, B. R. (1975). Studies of in vitro infection by $T$. cruzi. I. Ultrastructural studies on the invasion of macrophages and $\mathrm{L}$ cells. American Journal of Tropical Medicine and Hygiene 24, 25-33.

Weidemane, M.J., Smith, R., Hafney, T. \& Alaudeen, S. (1980). On the mechanism of the generation of chemiluminescence by macrophages. Behring Institut Mitteilungen 65, 42-54.

Wilson, C. B., Tsai, V. \& Remington, J. S. (1980). Failure to trigger the oxidative metabolic burst by normal macrophages. Possible mechanism for survival of intracellular pathogens. Journal of Experimental Medicine 151, 328-46. 